\title{
A novel predictor of clinical progression in patients on active surveillance for prostate cancer
}

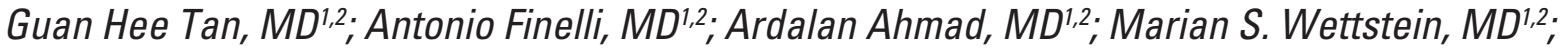

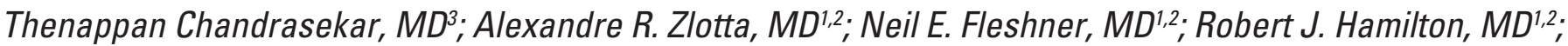

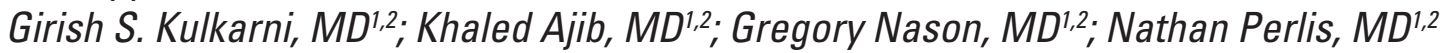

'Division of Urology, Princess Margaret Cancer Center and Toronto General Hospital, University Health Network, Toronto, ON, Canada; ${ }^{2}$ Division of Urology, University of Toronto, Toronto, ON, Canada; ${ }^{3}$ Department of Urology, Sidney Kimmel Cancer Center, Thomas Jefferson University, Philadelphia, PA, United States

Cite as: Can Urol Assoc J 2019;13(8):250-5. hitp://dx.doi.org/10.5489/cuaj.6122

\section{Abstract}

Introduction: Active surveillance (AS) is standard of care in low-risk prostate cancer (PCa). This study describes a novel total cancer location (TCLo) density metric and aims to determine its performance in predicting clinical progression (CP) and grade progression (GP). Methods: This was a retrospective study of patients on AS after confirmatory biopsy (CBx). We excluded patients with Gleason $\geq 7$ at $\mathrm{CBx}$ and $<2$ years followup. TCLo was the number of locations with positive cores at diagnosis (DBx) and CBx. TCLo density was TCLo/prostate volume (PV). CP was progression to any active treatment while GP occurred if Gleason $\geq 7$ was identified on repeat biopsy or surgical pathology. Independent predictors of time to CP or GP were estimated with Cox regression. Kaplan-Meier analysis compared progression-free survival (PFS) curves between TCLo density groups. Test characteristics of TCLo density were explored with receiver operating characteristic (ROC) curves.

Results: We included 181 patients who had CBx from 2012-2015 and met inclusion criteria. The mean age of patients was 62.58 years (standard deviation [SD] 7.13) and median followup was 60.9 months (interquartile range [IQR] 23.4). A high TCLo density score $(>0.05)$ was independently associated with time to $\mathrm{CP}$ (hazard ratio $[\mathrm{HR}]$ 4.70; 95\% confidence interval $[\mathrm{Cl}] 2.62-8.42 ; \mathrm{p}<0.001)$ and GP (HR 3.85; 95\% Cl 1.91-7.73; $\mathrm{p}<0.001)$. ROC curves showed TCLo density has greater area under the curve than number of positive cores at $\mathrm{CBx}$ in predicting progression.

Conclusions: TCLo density is able to stratify patients on AS for risk of $\mathrm{CP}$ and GP. With further validation, it could be added to the decision-making algorithm in AS for low-risk localized PCa.

\section{Introduction}

Active surveillance (AS) is the standard of care for low-risk localized prostate cancer (PCa). The major clinical guidelines recommend this approach, as it is known that local interventions aimed at eradicating cancer from the prostate gland are associated with urinary and/or sexual dysfunc- tion, and alterations to quality of life to varying extents. ${ }^{1-4}$ Therefore, the objective of AS is to help patients avoid potentially morbid treatments, which may be unnecessary for lowrisk localized PCa that carries minimal metastatic potential.

Unfortunately, a fair proportion of patients who initially go onto an AS program are destined to progress and require active treatment. One of the reasons for grade progression (GP) while on AS is that up to $30 \%$ of men who actually harbor highergrade cancer are misattributed as having low-grade cancer at initial biopsy. As it is thought that high-volume Gleason pattern 3 portends a greater risk of harboring aggressive cancer, ${ }^{5}$ most contemporary protocols include number of positive cores at biopsy and percentage core involvement as criteria for enrollment into an AS program. In addition, T-stage, Gleason score, prostate-specific antigen (PSA) and PSA density are also commonly considered when deciding whether or not a patient is suitable for AS. ${ }^{6-12}$ Despite all these criteria, $27 \%$ of the Sunnybrook series (median followup 6.4 years), ${ }^{13} 33 \%$ of the Johns Hopkins series (median followup 2.7 years), ${ }^{7}$ and $21 \%$ of the PRIAS cohort (median followup 1.6 years) still progressed to active intervention. ${ }^{10}$ Therefore, it remains a challenge to accurately identify the right patients for AS.

Recently, cumulative cancer location (CCLO) was described as a good predictor of disease progression in patients on AS. This metric involves dividing the prostate gland into six locations and determining how many locations harbored cancer at initial diagnostic biopsy (DBx) and subsequent confirmatory biopsy (CBx). The authors demonstrated that the risk of progression increased if more locations were affected by cancer, and it was found to be a better predictor than number of positive cores at $\mathrm{CBx} .{ }^{14}$ We attempted to improve on the CCLO metric by accounting for all cancer locations instead of limiting it to six areas. We went a step further to create a novel metric called total cancer location (TCLo) density, by incorporating prostate volume (PV) into the equation, as it was noted that men with smaller PV were more likely to progress. 
We hypothesized that because TCLo density accounts for all the disease sites as well as PV, it would be a superior predictor of disease progression. This study aims to determine the accuracy of TCLo density in predicting risk for clinical progression (CP) to receive active treatment, and/or GP in patients on AS for prostate cancer.

\section{Methods}

Approval was obtained from the Research and Ethics Board of University Health Network (08-0472). This was a retrospective study of patients who were continued on AS after CBx for T1 - T2a, PSA $\leq 15 \mathrm{ng} / \mathrm{mL}$, Gleason $6(3+3)$ prostate cancer. We excluded patients with Gleason 7 or higher at $\mathrm{CBx}$, less than two years followup, and incomplete data. TCLo was the sum of the number of locations with positive cores at DBx and CBx. This was calculated based on pathological description of where the positive biopsy cores were located. Theoretically, the TCLo model allows for an unlimited number of total locations because each core could represent a discrete location, so long as the positive cores were found in distinct parts of the prostate. However, if two or more cores were found in one particular area, for example the left lateral base, then it would only be counted as one location in the TCLo calculation. If positive cores were found in the same location at both DBx and CBx, the TCLo metric would consider it as a single location in the final summation. We then calculated the TCLo density determined by the function TCLo/PV.

We defined $\mathrm{CP}$ as progression to any active treatment, whereas GP occurred if Gleason 7 or higher was identified on repeat biopsy or surgical pathology. Univariate analyses with t-test and Mann-Whitney U-tests were used to compare characteristics of patients who had CP or GP vs. those who did not progress. Independent predictors of time to $\mathrm{CP}$ or GP were estimated with Cox regression using TCLo, TCLo density, number of positive $\mathrm{CBx}$ cores, percentage of positive cores at $\mathrm{CBx}$, number of positive DBx cores, and percentage of positive cores at DBx as predictors. These were adjusted for interactions with age and PSA. Kaplan-Meier analysis compared progression-free survival (PFS) curves between high $(>0.05)$ and low $(\leq 0.05)$ TCLo density groups. Test characteristics of TCLo density were explored with receiver operating characteristic (ROC) curves. A p-value of 0.05 or less was considered significant. All analyses were performed using the IBM ${ }^{\circledR}$ SPSS $^{\circledR}$ Statistics version 25 software.

\section{Results}

From 2012-2015, 421 patients had a CBx while being on AS for PCa. We included 181 patients who met inclusion criteria. The mean age of patients at CBx was 62.58 years (standard deviation [SD] 7.13) and the median PSA at diag- nosis was $5.16 \mathrm{ng} / \mathrm{mL}$ (interquartile range [IQR] 3.44). The median PV of this cohort was $41.0 \mathrm{~mL}$ (IQR 22.5). They were followed up for a median duration of 60.9 months (IQR 23.4). The median TCLo density was 0.049 (IQR 0.060). The other patient characteristics are shown in Table 1.

Sixty-nine $(38.1 \%)$ patients progressed to receive active treatment during AS. The majority of patients (56.5\%) went on to have radical prostatectomy as the definitive treatment. The other forms of treatment were external beam radiotherapy, brachytherapy, high-intensity focused ultrasound (HIFU), and watchful waiting. The variables that were significantly associated with progression to active treatment on univariate analysis were younger age, smaller PV, greater TCLo and TCLo density, higher percentage of positive cores at DBx and $\mathrm{CBx}$, and more positive cores at $\mathrm{CBx}$ (Table 2). Fortysix $(25.4 \%)$ patients were found to have GP while being on AS. Smaller PV, higher TCLo, TCLo density, percentage of positive cores at $\mathrm{DBx}$ and $\mathrm{CBx}$, and a greater number of positive cores at $\mathrm{CBx}$ were also significantly related to GP in univariate analysis (Table 2).

Cox regression analysis showed TCLo density was the strongest predictor for time to CP and GP among all the variables tested. A high TCLo density $(>0.05)$ was independently associated with time to CP (hazard ratio [HR] 4.70; $95 \%$ confidence interval $[\mathrm{Cl}] 2.62-8.42 ; \mathrm{p}<0.001)$ and GP (HR 3.85; 95\% Cl 1.91-7.73; $\mathrm{p}<0.001)$. The other variables that were associated with time to CP and GP were TCLo, number of positive cores at $\mathrm{CBx}$, and percentage positive cores at CBx and DBx. The number of positive cores at DBx was not a predictor for CP or GP (Table 3). The TCLo density also demonstrated good predictive characteristics in several other analyses. For example, men with high TCLo density $(>0.05)$ had significantly lower PFS rates for CP and GP (Fig. 1). Additionally, TCLo density outperformed number of positive cores at $\mathrm{CBx}$ in predicting $\mathrm{CP}$ and $\mathrm{GP}$, as shown by the ROC curves in Fig. 2.

\begin{tabular}{lc}
\hline Table 1. Patient characteristics & \\
\hline Variable & $\begin{array}{c}\text { All patients, } \\
\mathbf{n = 1 8 1}\end{array}$ \\
\hline Age at CBx, year, mean (SD) & $62.58(7.13)$ \\
PSA at diagnosis, $\mathrm{ng} / \mathrm{mL}$, median (IQR) & $5.16(3.44)$ \\
Prostate volume, $\mathrm{mL}$, median (IQR) & $41.00(22.50)$ \\
Number of cores taken at confirmatory biopsy, & $16(3.0)$ \\
median (IQR) & \\
Percentage of diagnostic cores at confirmatory & $11.76(17.42)$ \\
biopsy, median (IQR) & $2(2.0)$ \\
TCLo, n, median (IQR) & $0.049(0.060)$ \\
TCLo density, median (IQR) & $69(38.1 \%)$ \\
Progressed to active treatment while on AS, n (\%) & $46(25.4 \%)$ \\
Grade progression while on AS, $\mathrm{n}$ (\%) & \\
\hline AS: active surveillance; CBx: confirmatory biopsy; IOR: interquartile range; PSA: prostate- \\
specific antigen; SD: standard deviation; TCLo: total cancer locations. \\
\hline
\end{tabular}




\begin{tabular}{|c|c|c|c|c|c|c|}
\hline \multirow[t]{2}{*}{ Variable } & \multicolumn{2}{|c|}{ Progressed to active treatment } & \multirow[t]{2}{*}{$\mathbf{p}$} & \multicolumn{2}{|c|}{ Grade progression } & \multirow[t]{2}{*}{$\mathbf{p}$} \\
\hline & $\begin{array}{c}\text { Yes } \\
n=69\end{array}$ & $\begin{array}{c}\text { No } \\
n=112\end{array}$ & & $\begin{array}{c}\text { Yes } \\
n=46\end{array}$ & $\begin{array}{c}\text { No } \\
n=135\end{array}$ & \\
\hline Age at $\mathrm{CBx}$, year, mean (SD) & $60.97(6.15)$ & $63.57(7.53)$ & 0.017 & $62.26(5.76)$ & $62.69(7.56)$ & 0.690 \\
\hline PSA at diagnosis, ng/mL, median (IQR) & $5.04(2.48)$ & $5.24(3.71)$ & 0.390 & $5.45(2.36)$ & $5.08(3.64)$ & 0.609 \\
\hline Prostate volume, $\mathrm{mL}$, median (IOR) & $36.00(18.00)$ & $44.00(22.73)$ & $<0.001$ & $36.50(21.25)$ & $43.00(22.00)$ & 0.024 \\
\hline No. of cores taken at confirmatory biopsy, $n$, median (IQR) & $17.0(3.5)$ & $16.0(2.0)$ & 0.929 & $16.5(3.0)$ & $16.0(2.0)$ & 0.718 \\
\hline TCLo, median (IQR) & $3(2)$ & $2(2)$ & $<0.001$ & $3(2)$ & $2(2)$ & 0.004 \\
\hline TCLo density, median (IQR) & $0.078(0.067)$ & $0.038(0.035)$ & $<0.001$ & $0.071(0.066)$ & $0.045(0.053)$ & $<0.001$ \\
\hline Number of positive cores at $\mathrm{CBx}, \mathrm{n}$, median (IQR) & $3(4)$ & $1(2)$ & $<0.001$ & $3(3)$ & $1(2)$ & 0.020 \\
\hline Percentage of positive cores at $\mathrm{CBx}, \%$ median (IQR) & $17.65(19.15)$ & $7.69(9.91)$ & $<0.001$ & $16.67(21.42)$ & $10.53(12.87)$ & 0.026 \\
\hline Number of positive cores at DBx, n, median (IQR) & $2(1)$ & $1(1)$ & 0.141 & $2(1)$ & $1(1)$ & 0.074 \\
\hline Percentage of positive cores at $\mathrm{DBx}, \%$ median (IQR) & $14.29(11.48)$ & $10.00(8.97)$ & 0.001 & $15.38(11.67)$ & $10.00(8.81)$ & 0.002 \\
\hline
\end{tabular}

CBx: confirmatory biopsy; DBx: diagnostic biopsy; IQR: interquartile range; PSA: prostate-specific antigen; SD: standard deviation; TCLo: total cancer locations.

\section{Discussion}

Most of the major contemporary AS series have slightly different entry criteria. ${ }^{6}$ While they all consider clinical T stage, Gleason score, and number of positive biopsy cores for enrollment into an AS program, not all protocols place particular importance on PSA, PSA density, and percentage core involvement when determining patient eligibility for AS..$^{7-13}$ Although some protocols have more stringent entry criteria than others, they do not always translate into better clinical outcomes. As one study had shown, a stricter protocol for AS does not seem to produce better end results than a more inclusive protocol. ${ }^{15}$ Furthermore, having overly restrictive criteria would mean denying more patients from the benefits of AS. We are certainly in need of better predictive methods.
The concept of evaluating the cumulative number of cancer locations at DBx and CBx (CCLO), rather than just the absolute number of positive cores at $\mathrm{CBx}$ in relation to the risk of progression is interesting. It gives us an idea of how extensively distributed the cancer is within the prostate gland, and the hypothetical implication being patients with greater number of locations involved with cancers are at higher risk of progression while on AS. Erickson et al were able to demonstrate in their study that a greater CCLO was associated with higher risk of progression and it outperformed number of positive cores at $\mathrm{CBx}$ in predicting the event. ${ }^{14}$

We initially attempted to apply and reproduce the CCLO metric on our study population. However, the CCLO has a rigid method of calculation, as it divides the prostate into only six locations. This limits its potential to truly reflect the extent and distribution of cancer within the prostate gland.

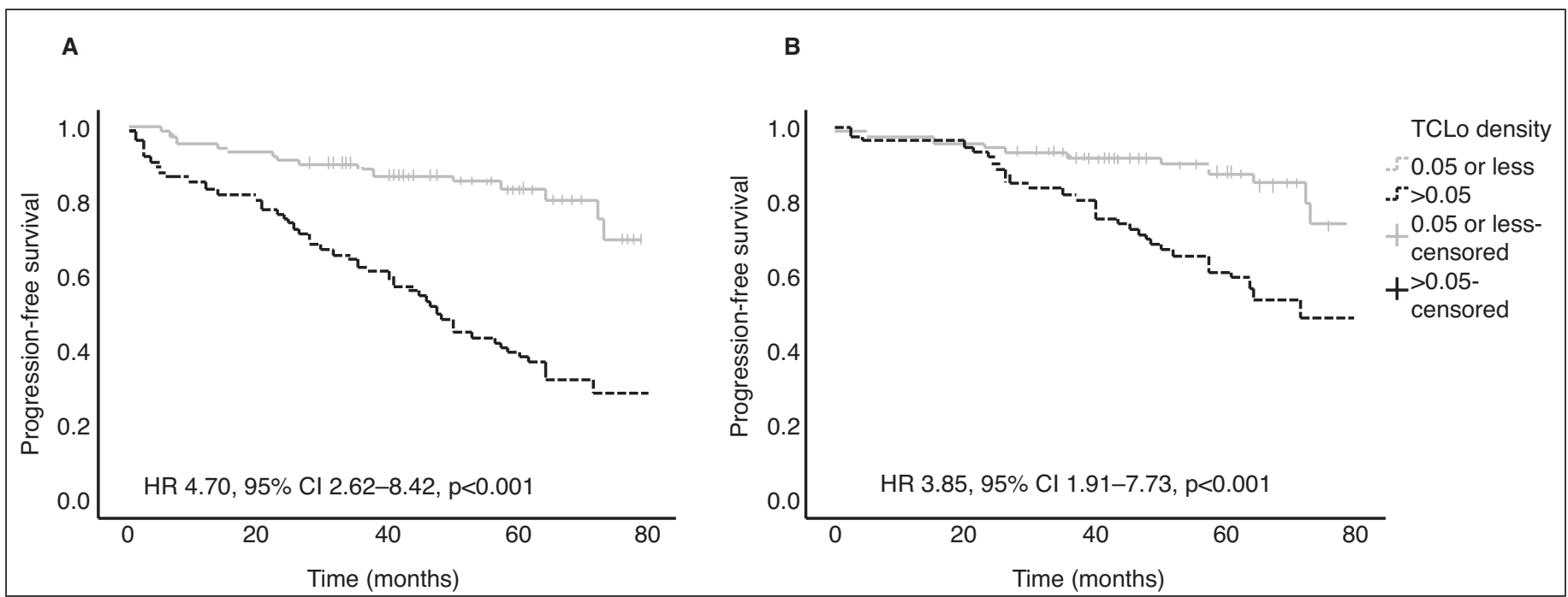

Fig. 1. Kaplan-Meier curves comparing progression-free survival (PFS) in patients with high $(>0.05)$ or low $(\leq 0.05)$ total cancer location (TCLo) density. The curves for $(\boldsymbol{A})$ progression to active treatment and (B) grade progression demonstrate poorer PFS in patients with high TCLo density. Cl: confidence interval; HR: hazard ratio. 


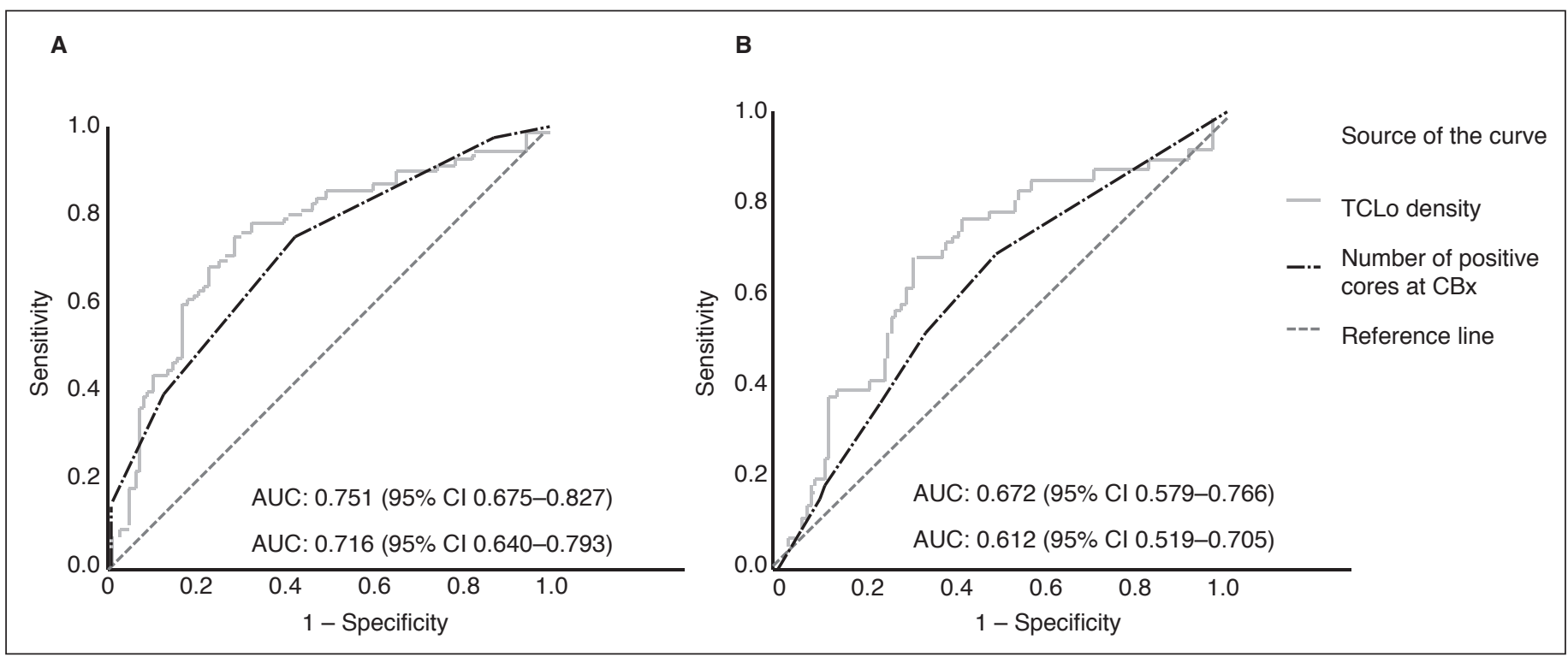

Fig. 2. Receiver operating characteristics (ROC) curves comparing total cancer location (TCLo) density and number of positive cores at confirmatory biopsy (CBx). The ROC curves for ( $\boldsymbol{A}$ ) progression to active treatment and $(\boldsymbol{B})$ grade progression demonstrate that TCLo density has greater area under the curve (AUC) than number of positive cores at CBx. Cl: confidence interval.

Additionally, our institution has been labeling the source of the biopsy cores more precisely in recent years. For example, instead of labeling just apex, mid-gland, and base in each prostate lobe, it is now refined into apex, mid-gland, and base of the medial and lateral aspect in each lobe. This means the prostate is divided into 12 distinct locations. Furthermore, some patients are subjected to additional targeted cores based on information from multiparametric magnetic resonance imaging (mpMRI) of their prostate glands. Our TCLo model considers these as distinct locations in the final summation of TCLo if they were not within the area of systematic samples. Therefore, we took an approach of including an unlimited number of locations for calculation of TCLo, and essentially sum up the number of locations based on where the positive biopsy cores were taken (Fig. $3)$. Next, we made an observation that men with smaller PV tended to progress more often than those with larger PV despite having the same TCLo. Univariate analysis showed the median PV of men who progressed was indeed smaller
(Table 2). This brought about the idea of combining PV with TCLo to produce a novel density metric, similar to the concept of PSA density.

As was mentioned, most AS protocols take into account the number of positive biopsy cores when enrolling patients for AS. ${ }^{6}$ It is also a clinical parameter that is considered for patients to remain on AS. For example, the PRIAS, Johns Hopkins, and University of Miami protocols would only enroll patients with no more than two positive cores for AS, ${ }^{7,10-12}$ whereas the Memorial Sloan Kettering Cancer Center protocol allows for up to three positive cores. ${ }^{8}$ Although there are slight variations, it is generally accepted that the number positive biopsy cores does influence our clinical decision.

With this in mind, we set out to compare the performance of TCLo density against number of positive cores at CBx in predicting CP and GP. This study demonstrated that TCLo density outperformed number of positive cores at $\mathrm{CBx}$ in predicting $\mathrm{CP}$ and $\mathrm{GP}$, and men with high TCLo density

\begin{tabular}{|c|c|c|c|c|}
\hline \multirow[t]{2}{*}{ Variable } & \multicolumn{2}{|c|}{ Progressed to treatment } & \multicolumn{2}{|c|}{ Grade progression } \\
\hline & HR (95\% Cl) & $\mathbf{p}$ & HR (95\% Cl) & $\mathbf{p}$ \\
\hline TCLo & $1.64(1.38-1.96)$ & $<0.001$ & $1.32(1.08-1.62)$ & 0.007 \\
\hline TCLo density $(>0.05)$ & $4.70(2.62-8.42)$ & $<0.001$ & $3.85(1.91-7.73)$ & $<0.001$ \\
\hline Number of positive cores at $\mathrm{CBx}$ & $1.48(1.31-1.67)$ & $<0.001$ & $1.20(1.04-1.39)$ & 0.012 \\
\hline Percentage of positive cores at $\mathrm{CBx}$ & $1.06(1.04-1.08)$ & $<0.001$ & $1.03(1.01-1.05)$ & 0.007 \\
\hline Number of positive cores at DBx & $1.18(0.86-1.61)$ & 0.301 & $1.31(0.89-1.92)$ & 0.169 \\
\hline Percentage of positive cores at DBx & $1.02(1.00-1.04)$ & 0.026 & $1.03(1.01-1.05)$ & 0.002 \\
\hline
\end{tabular}

CBx: confirmatory biopsy; Cl: confidence interval; DBx: diagnostic biopsy; HR: hazard ratio; PSA: prostate-specific antigen; TCLo: total cancer locations. 


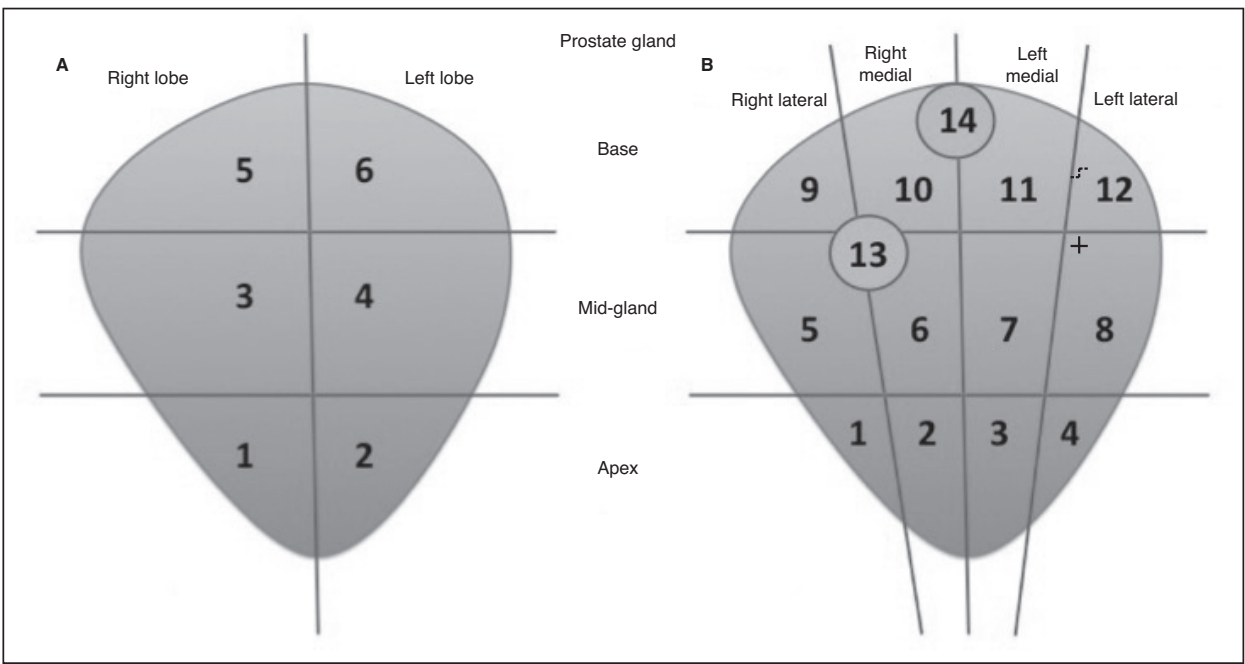

Fig. 3. Comparison between how CCLO and TCLo are calculated. (A)CCLO is limited to a maximum of six cancer locations, while (B) TCLo sums up the total number of cancer locations according to the location that has been labeled by the biopsy operator. In this example, the right and left prostate lobes are further divided into medial $(2,3,6,7,10,11)$ and lateral $(1,4,5,8,9,12)$ locations. TCLo would also count targeted cores $(13,14)$ as distinct cancer locations.

defined as $>0.05$ had poorer PFS (Figs. 1, 2). It is also the strongest independent predictor for CP and GP among the variables we tested (Table 3).

Numerous research efforts are underway to improve our ability to select the most suitable patients for AS. The two areas that are intensely being studied are development of predictive biomarkers and utility of mpMRI. Biomarkers that have been studied for predicting progression of disease in AS include Prostate Health Metric (PHI), prostate cancer antigen 3 (PCA3), TMPRSS2:ERG, and the Decipher Test. ${ }^{16-21}$ While they have mostly shown positive association with progression and higher-grade cancer, there have also been negative results, such as in one study on PCA3. ${ }^{22}$ To date, these biomarkers have yet to find their place in AS for prostate cancer. The use of mpMRI in the context of AS is being widely investigated as well. Bloom et al demonstrated that a negative CBx with MRI-ultrasound fusion biopsy is associated with reduced risk of grade group progression (HR $0.41 ; 95 \% \mathrm{Cl} 0.22-0.77 ; \mathrm{p}<0.01) .{ }^{23}$ Others have used mpMRI as a followup tool and they found that performing serial scans add incremental value to just cancer core length at baseline biopsy and PSA density for predicting upgrading in patients on AS. ${ }^{24}$ Another study created a nomogram combining MRI information with clinical parameters and that has been shown to predict progression more accurately. ${ }^{25}$ Again, as with biomarkers, the exact role of mpMRI is still undefined in AS.

While the results of studies on such novel predictive tests are impressive and promising, they are not always available for general use and could even potentially be very costly. We have shown TCLo density to be more robust than number of positive biopsy cores at CBx in predicting progression. Furthermore, it does not incur any extra cost, as it uses information that is readily available at DBx and CBx. Therefore, adding TCLo density to our current algorithm in the management of AS seems practical and economical.

We accept that there are limitations to our study. The retrospective nature of this study exposes it to inherent selection bias. A fair proportion of patients had to be excluded, as they did not have adequate information in our hospital records. Every effort was made to be as accurate as possible in the data collection process. We recognize that TCLo density needs further validation and must be replicable in other AS populations. Nonetheless, the results of this study are very encouraging and we might now have a new metric that could help us in the management of PCa patients on AS.

\section{Conclusions}

The TCLo density is able to stratify patients on AS for PCa into low- or high-risk for CP and GP. It is a stronger predictor of progression than number of positive cores at $\mathrm{CBx}$, which is a common entry criteria into an AS program. While it needs further validation, it has the potential to be a part of the decision-making algorithm in AS for low-risk localized PCa.

Competing interests: Dr. Finelli has been an advisory board member for Abbvie, Astellas, Bayer, Ipsen, Janssen, Sanofi, and TerSera; and has participated in clinical trials supported by Astellas, Bayer, and Janssen. Dr. Zlotta has been an advisory board member for Janssen, Rho Inc, and Sanofi. Dr. Fleshner has been a consultant or advisory board member for Abbvie, Amgen, Astellas, Bayer, Ferring, Hybridyne Health, Janssen, and Sanofi; and has participated in clinical trials supported by Astellas, Bavarian Nordic, Bayer, Ferring, Janssen, Medivalion, Nucleix, Progenics Pharmaceutical, Sanofi, and Spectracure AB. Dr. Perlis has been an advisory board member for and received honoraria from TerSera; holds shares in Focal Healthcare; and participated in a clinical trial supported by Insightec. The remaining authors report no competing personal or financial interests related to this work. 


\section{References}

1. Mottet N, van den Bergh RCN, Briers E, et al. EAU-ESTRO-ESUR-SIOG guidelines on prostate cancer. Available at: http://uroweb.org/wp-content/uploads/EAU-Guidelines-2018-compilation-of-all-guidelines. pdf. Accessed Feb. 28, 2019.

2. Sanda $M$, Chen R, Crispino $T$, et al. Clinically localized prostate cancer: AUA/ ASTRO/SUO guideline. Available at: https://www.auanet.org/guidelines/prostate-cancer-clinically-localized-(2017). Accessed Feb. 28, 2019.

3. Penson DF, Rossignol M, Sartor A0, et al. Prostate cancer: Epidemiology and health-related quality of life. Urology 2008;72:S3-11. htrps://doi.org/10.1016/i.urology.2008.10.006

4. Sureda A, Fumadó $L$, Ferrer $M$, et al. Health-related quality of life in men with prostate cancer undergoing active surveillance vs. radical prostatectomy, external-beam radiotherapy, prostate brachytherapy and reference population: A cross-sectional study. Health Qual Life Outcomes 2019;17:11. https://doi.org/10.1186/s12955-019-1082-4

5. Perlis N, Klotz L. Contemporary active surveillance: Candidate selection, followup tools, and expected outcomes. Urol Clin North Am 2017;44:565-574. hitps://doi.org/10.1016/j.ucl.2017.07.005

6. Chung MS, Lee SH. Current status of active surveillance in prostate cancer. Investig Clin Urol 2016;57:1420. https://doi.org/10.4111/icu.2016.57.1.14

7. Tosoian JJ, Trock BJ, Landis P, et al. Active surveillance program for prostate cancer: An update of the Johns Hopkins experience. J Clin Oncol 201 1;29:2185-90. https://doi.org/10.1200/JC0.2010.32.8112

8. Adamy A, Yee DS, Matsushita K, et al. Role of prostate specific antigen and immediate confirmatory biopsy in predicting progression during active surveillance for low-isk prostate cancer. J Urol 2011;185:477-82. https://doi.org/10.1016/i.juro.2010.09.095

9. Whitson JM, Porten SP, Hilton JF, et al. The relationship between prostate-specific antigen change and biopsy progression in patients on active surveillance for prostate cancer. J Urol 2011;185:1656-60. https://doi.org/10.1016/i.juro.2010.12.042

10. Bul M, Zhu X, Valdagni R, et al. Active surveillance for low-risk prostate cancer worldwide: The PRIAS study. Eur Urol 2013;63:597-603. https://doi.org/10.1016/i.eururo.2012.11.005

11. Soloway MS, Soloway CT, Eldefrawy A, et al. Careful selection and close monitoring of low-risk prostate cancer patients on active surveillance minimizes the need for treatment. Eur Urol 2010;58:831-5. https://doi.org/10.1016/i.eururo.2010.08.027

12. van den Bergh RC, Roemeling S, Roobol MJ, et al. Outcomes of men with screen-detected prostate cancer eligible for active surveillance who were managed expectantly. Eur Urol 2009;55:1-8. https://doi.org/10.1016/i.eururo.2008.09.007

13. Klotz L, Vesprini $D$, Sethukavalan $P$, et al. Long-term followup of a large active surveillance cohort of patients with prostate cancer. J Clin Oncol 2015;33:272-7. https://doi.org/10.1200/JC0.2014.55.1192
14. Erickson AM, Luzzagod S, Semionowf A, et al. Cumulative cancer locations is a novel metric for predicting active surveillance outcomes: A multicenter study. Eur Urol Oncol 2018;1:268-75. https://doi.org/10.1016/i.euo.2018.04.006

15. Komisarenko M, Timilshina N, Richard PO, et al. Stricter active surveillance criteria for prostate cancer do not result in significantly better outcomes: A comparison of contemporary protocols. J Urol 2016;196:1645-50. https://doi.org/10.1016/i.juro.2016.06.083

16. Moschini M, Carroll PR, Eggener SE, et al. Low-risk prostate cancer: Identification, management, and outcomes. Eur Urol 2017;72:238-49. https://doi.org/10.1016/i.eururo.2017.03.009

17. Kim JH, Hong SK. Potential utility of novel biomarkers in active surveillance of low-risk prostate cancer. Biomed Res Int 2015;2015:475920. hittps://doi.org/10.1155/2015/475920

18. Tosoian JJ, Loeb S, Feng Z, et al. Association of [-2]proPSA with biopsy reclassification during active surveitlance for prostate cancer. J Urol 2012;188:1131-6. https://doi.org/10.1016/i.juro.2012.06.009

19. Lin DW, Newcomb LF, Brown EC, et al. Urinary TMPRSS2:ERG and PCA3 in an active surveillance cohort: results from a baseline analysis in the Canary Prostate Active Surveillance Study. Clin Cancer Res 2013;19:2442-50. https://doi.org/10.1158/1078-0432.CCR-12-3283

20. Berg KD, Vainer $B$, Thomsen $F B$, et al. ERG protein expression in diagnostic specimens is associated with increased risk of progression during active surveillance for prostate cancer. Eur Urol 2014;66:851-60. https://doi.org/10.1016/i.eururo.2014.02.058

21. Kim HL, Li P, Huang HC, et al. Validation of the Decipher Test for predicting adverse pathology in candidates for prostate cancer active surveillance. Prostate Cancer Prostatic Dis 2018 Dec 12. [Epub ahead of print]. https://doi.org/10.1038/s41391-018-0101-6

22. Tosoian JJ, Loeb S, Kettermann A, et al. Accuracy of PCA3 measurement in predicting short-term biopsy progression in an active surveillance program. J Urol 2010;183:534-8. https://doi.org/10.1016/i. juro.2009.10.003

23. Bloom JB, Hale GR, Gold SA, et al. Predicting Gleason group progression for men on prostate cancer active surveillance: Role of a negative confirmatory magnetic resonance imaging-ultrasound fusion biopsy. J Urol 2019;201:84-90. https://doi.org/10.1016/i.juro.2018.07.051

24. Felker ER, Wu J, Natarajan $\mathrm{S}$, et al. Serial magnetic resonance imaging in active surveillance of prostate cancer: Incremental value. J Urol 2016;195:1421-7. https://doi.org/10.1016/i.juro.2015.11.055

25. Lai WS, Gordetsky JB, Thomas JV, et al. Factors predicting prostate cancer upgrading on magnetic resonance imaging-targeted biopsy in an active surveillance population. Cancer 2017;123:1941-8. https://doi.org/10.1002/cncr.30548

Correspondence: Dr. Guan Hee Tan, Division of Urology, Princess Margaret Cancer Center and Toronto General Hospital, University Health Network, Toronto, ON, Canada; mrtan.guanhee@gmail.com 